\title{
Mutations in variable domains of the HIV-1 envelope gene can have a significant impact on maraviroc and vicriviroc resistance
}

\author{
Odalis Asin-Milan ${ }^{1,2}$, Annie Chamberland ${ }^{1,2}$, Yi Wei $^{1}$, Alpha Haidara ${ }^{1,2}$, Mohamed Sylla1,2 \\ and Cécile L Tremblay ${ }^{1,2,3,4^{*}}$
}

\begin{abstract}
Background: Resistance to CCR5 inhibitors, such as maraviroc and vicriviroc is characterized by reduction of maximal percent inhibition which indicates the use of an inhibitor-bound conformation of CCR5 for human immunodeficiency virus-1(HIV-1) entry. It is accompanied by substitutions in gp120 and gp41. Variable domain 3 (V3) plays the most important role, but substitutions outside $\mathrm{V} 3$ could also be involved in phenotype resistance. In this work, we investigated how mutations in variable regions of the viral envelope protein gp120 can contribute to CCR5 inhibitor resistance.

Methods: Resistant isolates were selected by passaging CC1/85 and BaL viruses with sub-inhibitory MVC and VCV concentrations. Mutations in gp160 were identified and mutants containing V2 (V169M), V3 (L317W) and V4 (I408T) were constructed.

Results: MVC and VCV susceptibility and viral tropism were assessed by single cycle assay. Mutant 1408T showed 4-fold change $(F C)$ increase in the half maximal inhibitory concentration $\left(I C_{50}\right)$ to MVC, followed by L317W (1.52-FC), V169M (1.23-FC), V169M/1408T (4-FC) L317W/I408T (3-FC), V169M/L317W (1.30-FC), and V169M/L317W/I408T (3.31-FC). MPI reduction was observed for mutants I408T (85\%), L317W (95\%), V169M/I408T (84\%), L317W/I408T (85\%) and V169M/L317W/I408T (83\%). For VCV, I408T increased the IC 50 by 2-FC and few mutants showed MPI reduction less than 95\%: I408T (94\%), L317W/I408T (94\%) and V169M/L317W/I408T (94\%). All mutants remained R5-tropic and presented decreased infectivity.
\end{abstract}

Conclusions: These results suggest that mutations in the V4 loop of HIV-1 may contribute to MVC and VCV resistance alone or combined with mutations in V2 and V3 loops.

Keywords: Maraviroc, Vicriviroc, Resistance, CCR5 Inhibitors

\section{Background}

HIV-1 entry into target cells is initiated by interactions between the viral envelope (Env) protein gp120 and the host cell receptor CD4. It triggers conformational changes in gp120, forming the co-receptor binding site [1-3]. gp120 interaction with $\mathrm{C}-\mathrm{C}$ chemokine receptor 5 (CCR5) or C-X-C chemokine receptor 4 (CXCR4) induces other conformational changes in gp120, which

\footnotetext{
* Correspondence: c.tremblay@umontreal.ca

${ }^{1}$ Centre de recherche, Centre hospitalier de l'Université de Montréal (CRCHUM), Montréal, QC, Canada

2Department of Microbiology and Immunology, Faculty of Medicine, Université de Montréal, Montréal, QC, Canada

Full list of author information is available at the end of the article
}

evoke structural re-arrangement of gp41 and enables the viral and cellular membrane fusion, permitting viral entry [4]. CCR5 inhibitors, including maraviroc (MVC), vicriviroc (VCV), aplaviroc, TAK-779 and TAK-220, antagonize this process and have strong anti-viral activity against HIV-1 in vitro [5,6]. Although they bind the hydrophobic pocket within transmembrane domains of CCR5 with high affinity, they occupy different subcavities by interacting with different amino acids [6]. MVC is the first CCR5 inhibitor approved for the treatment of R5-tropic HIV-1 infection in both naïve and treatment-experienced adult patients. VCV development was stopped because of suboptimal efficacy [5]. Since
C Biomed Central 
MCV and VCV are allosteric inhibitors of virus entry, resistance to these drugs is evidenced by reduction in the plateau of virus inhibition curves rather than by increases in 50 percent inhibitory concentration $\left(\mathrm{IC}_{50}\right)$ $[7,8]$. The magnitude of this decrease can be expressed as maximum plateau inhibition (MPI) [9]. Plateau height depends on the relative affinity of HIV-1 for inhibitorbound versus free CCR5, the greater the affinity for inhibitor-bound CCR5, the lower the height of the plateau [7]. MOTIVATE clinical trials of MVC revealed that the MPI of most MVC-resistant viruses in subjects failing therapy ranged from 80 to $95 \%$ [9]. In VICTOR-E1 clinical trials of VCV, phenotypic resistance was manifested by reductions in relative MPI. The cut-off value was 0.94 [8]. Changes in susceptibility to CCR5 inhibitors are usually accompanied by substitutions in gp120, with V3 domain appearing to play a critical role. However, substitutions outside this region also contribute to the resistance phenotype [10]. The aim of this study is to investigate how mutations in other variable loops of the HIV-1 Env can contribute to MVC and VCV resistance.

\section{Results}

\section{Selection of resistance mutations by in vitro passage}

After 4 passages of $\mathrm{CC} 1 / 85$ virus in the presence of sub-inhibitory MVC concentrations, some mutations, such as V169M and N192K in V2, L317W in V3, I408A in V4, D462N, N463T, S464T and N465aD in V5, and L820I, I829V and Y837C in gp41,were associated with increased p24 levels (Table 1). After 16 passages, 2 new mutations materialized: I408T in V4 and P849Q in gp41 (Table 1). It is noteworthy that the mutation I408A in $\mathrm{V} 4$ appeared after 4 passages and disappeared at passage 16 , when a new mutation, I408T, surfaced at the same position.. For VCV, 2 mutations emerged after 4 passages: V169M in V2 and L317W in V3. After 16 passages, the mutation I840Y arose in gp41. No amino acid change was observed with either drug in the Env region of laboratory-adapted $\mathrm{BaL}$ virus after 16 passages (Additional file 1).

\section{Susceptibility of mutant clones to MVC and VCV}

Significant (85\%) reduction in MVC's MPI was apparent for $\mathrm{I} 408 \mathrm{~T}, 84 \%$ for the double mutant V169M/I408T, $85 \%$ for $\mathrm{L} 317 \mathrm{~W} / \mathrm{I} 408 \mathrm{~T}$, and $83 \%$ for the triple mutant V169M/L317W/I408T. L317W and V169M/L317W mutants presented intermediate resistance of $95 \%$ and $94 \%$, respectively. No significant MPI diminution occurred for the single mutant V169M (96\%). A similar pattern was observed when measuring fold-change (FC) increase in the $\mathrm{IC}_{50}$, with $\mathrm{I} 408 \mathrm{~T}$ showing 4-FC, L317W 1.52-FC, and $\mathrm{V} 169 \mathrm{M}, 1.23-\mathrm{FC}$ increase. The double mutants V169M/L317W exhibited 1.30-FC, V169M/I408T 4-FC and L317W/I408T 3-FC, and 3.31-FC was evident for the triple mutant V169M/L317W/I408T (Table 2 and Figure 1A). The single mutants V169M and L317W and the double mutants V169M/L317W and V169M/I408T retained their susceptibility to $\mathrm{VCV}$, reaching $100 \%$ inhibition in some cases. For VCV, I408T increased the $\mathrm{IC}_{50}$ by $2-\mathrm{FC}$, and the mutants $\mathrm{I} 408 \mathrm{~T}$, L317W/I408T and V169M/L317W/I408T showed MPI of 94\%, 93\% and $94 \%$, respectively (Table 3 and Figure 1B).

\section{Mutations affect virus infectivity differentially}

The CC1/85 parental clone and mutants exhibited different levels of infectivity. All mutants manifested decreased infectivity that ranged from 20 to $38 \%$. Infectivity percentage was $75 \%$ for V169M, 76\% for L317W, $80 \%$ for I408T, $76 \%$ for the double mutants V169M/L317W, 63\% for $\mathrm{V} 169 \mathrm{M} / \mathrm{I} 408 \mathrm{~T}$, $67 \%$ for L317W/I408T, and $67 \%$ for the triple mutant V169M/L317W/I408T (Figure 2).

\section{Tropism and V3 net charge}

All CC1/85-derived mutants remained R5-tropic. None of them was able to replicate in U87CD4CXCR4+ cells, but did so well in U87CD4CCR5+ cells (Tables 2 and 3). R5-tropism was also confirmed by 2 different genotypic predictors, PSSM $_{\mathrm{X} 4 \mathrm{R} 5}[11,12]$ and geno2pheno coreceptor [13]. No change in V3 net charge was observed.

\section{Discussion}

Our findings indicate that viruses resistant to MVC can retain the use of CCR5 coreceptor as reported previously [14-16]. CCR5 inhibitors are associated with mutations in the Env V3 region of R5 isolates [14,17]. Our passage experiments revealed only 1 polymorphism in the V3 loop crown, L317W, which was associated with reduced infectivity, but not with resistance to CCR5 inhibitors or changes in V3 net charge (Tables 1 and 2). The selection of HIV-1 resistance to CCR5 inhibitors is relatively difficult $[14,18]$, the V3 loop being the least variable of the HIV-1 Env variable regions [19]. Marozsan et al. found no amino acid changes in the $\mathrm{V} 3$ loop of $\mathrm{CC} 1 / 85$ resistant to VCV generated in vitro [15]. Wesby et al. reported a MVC-resistant $\mathrm{CC} 1 / 85$ virus generated in passage experiments with only 2 changes in amino acid positions 316 and 323 in the V3 loop [14]. Anastassopoulou et al. described D1/86.16, a VCV escape mutant that has no mutations in V3 [20].

Interestingly, when V3 substitution L317W is associated with V4 substitution I408T or triple substitution $\mathrm{V} 169 \mathrm{M} / \mathrm{L} 317 \mathrm{~W} / \mathrm{I} 408 \mathrm{~T}$, it confers further reduction of infectivity to $70 \%$ of the parental clone (Figure 2). This decreased infectivity could be due to lower fusion activity or binding affinity to the CCR5 co-receptor molecule of the mutant L317W.

The same polymorphism was found by $\mathrm{Yu}$ et al. in V3 region of the gp120 isolates CRF07_BC and CRF01_AE, 
Table 1 Summary of sequence changes in env gene associated with decreased susceptibility to MVC and VCV in passages experiments

\begin{tabular}{|c|c|c|}
\hline Virus, drug & Passage no. & gp 120 V2 loop amino acid sequence \\
\hline \multirow[t]{2}{*}{ CC1/85 } & start & 158 \\
\hline & & SFNITTSIRNKVQKQYALFYKLDWPIDNDSNNTNYRLISC \\
\hline CC1/85 control & 4 & ----- \\
\hline CC1/85, maraviroc & 4 & - \\
\hline CC1/85, vicriviroc & 4 & -M- \\
\hline CC1/85 control & 16 & \\
\hline $\mathrm{CC} 1 / 85$, maraviroc & 16 & 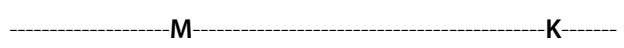 \\
\hline CC1/85, vicriviroc & 16 & 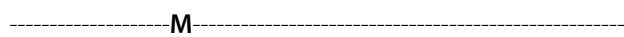 \\
\hline
\end{tabular}

gp 120 V3 loop amino acid sequence

CC1/85 start

296

CC1/85 control

CTRPNNYTRKSIHIGPGRALYATGDIIGDIRQAHC

CC1/85, maraviroc

CC1/85, vicriviroc

CC1/85 control

CC1/85, maraviroc

CC1/85, vicriviroc

gp 120 V4 loop amino acid sequence

\section{CC1/85 control}

CC1/85, maraviroc

CC1/85, vicriviroc

CC1/85 control

CC1/85, maraviroc

CC1/85 control

CC1/85, maraviroc

CC1/85, vicriviroc

CC1/85 control

CC1/85, maraviroc

CC1/85, vicriviroc

$\mathrm{CC} 1 / 85$

CC1/85 control

CC1/85, maraviroc

CC1/85, vicriviroc

CC1/85 control

CC1/85, maraviroc

CC1/85, vicriviroc
CNSTKLFNSTWTWNNSTWNNTKRANDIEEIITLPC

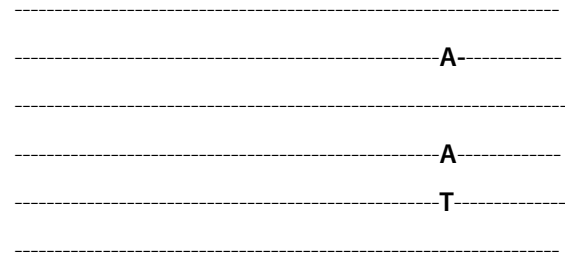

KDNSTNEIFR

TN--T---N--

TNTT-D----

gp 41 amino acid sequence

642

IYNLLEESQNQQEKNEQELLELDKWANLWNWFDISNWLWY 
Table 1 Summary of sequence changes in env gene associated with decreased susceptibility to MVC and VCV in passages experiments (Continued)

\begin{tabular}{llll}
\hline CC1/85 & & gp $\mathbf{4 1}$ amino acid sequence \\
& start & 812 \\
IKIFINATALAVAEGTDRILEVLQRAYRAILHIPRRIRP
\end{tabular}

Residues are numbered according to the HXB2 gp120 sequence.

the predominant HIV-1 subtypes in China in patients naïve to CCR5 inhibitors. In their study, R5 isolates harboring 2 dominant polymorphisms, R315Q and F317W in V3, were less susceptible than B isolates to MVC and TAK779. They postulated that baseline resistance to

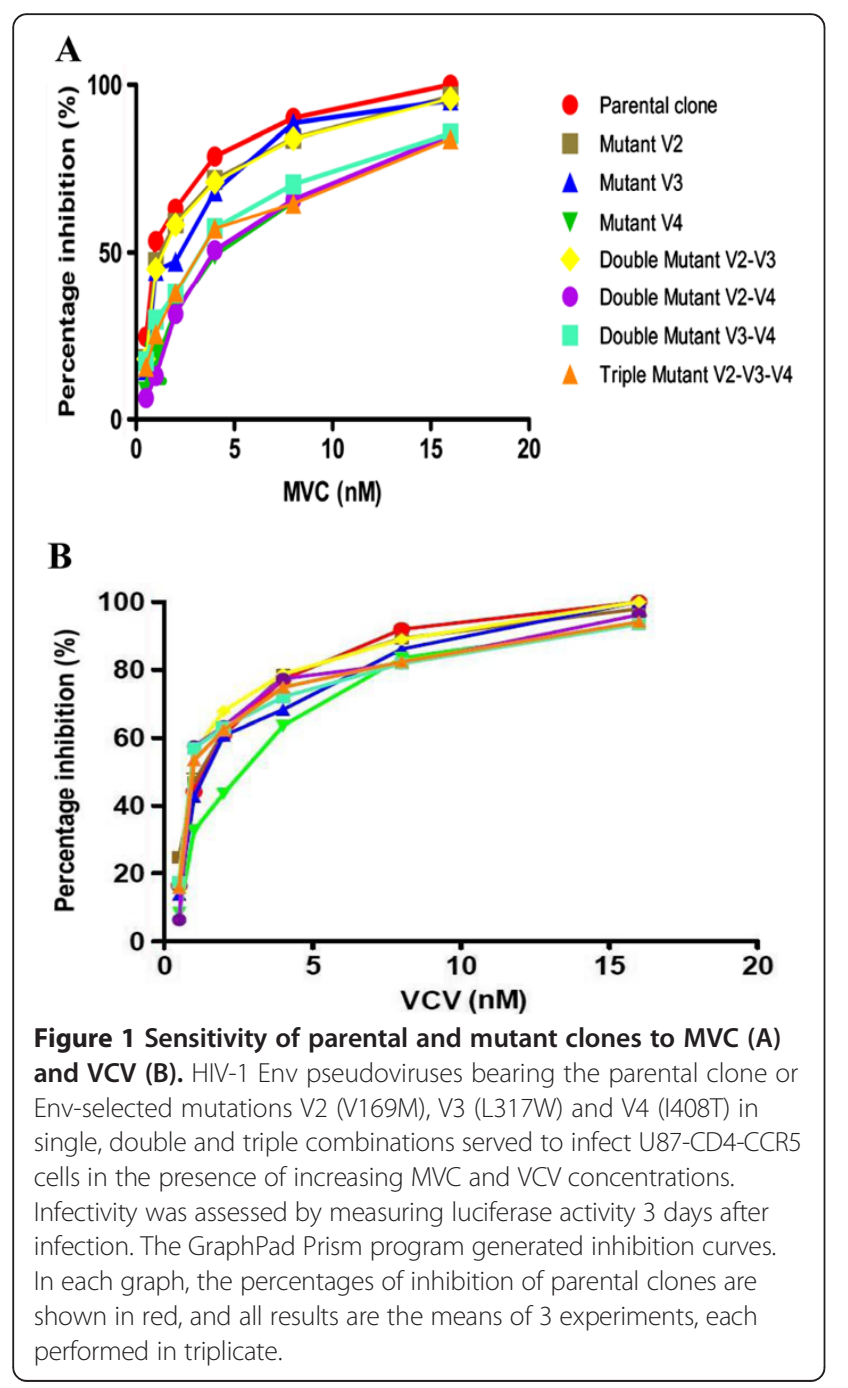

entry inhibitors may be associated with naturallyoccurring polymorphisms [21]. Yuan et al. [22] studied a combination of polymorphisms in the gp120 V3 loop of HIV-1JR-FL virus that can confer non-competitive resistance to MVC with a V3 loop library containing a set of random combinations of 0-10 polymorphic mutations in vitro. After 17 passages, they found pseudoviruses containing the polymorphism F317W (F312W from the V3 library) with typical non-competitive resistance to MVC. The polymorphism F317W was also found by Muñoz-Nieto et al. in 2008 in the env region from primary isolates during follow-up of dual natural infection with subtypes B and G [23].

In our study, I408T in V4 was the mutation that conferred the highest level of resistance. This mutation

Table 2 Susceptibility and phenotypic properties of V2, V3 and V4 single, double and triple mutants against maraviroc

\begin{tabular}{llllll}
\hline Loop & Mutant & Tropism & IC 50 & $\begin{array}{l}\text { IC } 50 \\
\text { FC increase }\end{array}$ \\
\hline Parental clone & Wild type & R5 & $1.47 \mathrm{nM}$ & - & - \\
V2 & V169M & R5 & $1.82 \mathrm{nM}$ & 1.23 & $96 \%$ \\
V3 & L317W & R5 & $2.24 \mathrm{nM}$ & 1.52 & $95 \%$ \\
V4 & I408T & R5 & $5.46 \mathrm{nM}$ & 4.00 & $85 \%$ \\
V2-V3 & V169M & R5 & $1.92 \mathrm{nM}$ & 1.30 & $94.3 \%$ \\
& L317W & & & & \\
V2-V4 & V169M & R5 & $5.76 \mathrm{nM}$ & 4.00 & $84 \%$ \\
& I408T & & & & \\
V3-V4 & L317W & R5 & $4.29 \mathrm{nM}$ & 3.00 & $85 \%$ \\
& I408T & & & & \\
V2-V3-V4 & V169M & R5 & $4.88 \mathrm{nM}$ & 3.31 & $83 \%$ \\
& L317W & & & & \\
& I408T & & & & \\
\hline
\end{tabular}

$\mathrm{IC}_{50}$, 50\% effective concentration or concentration needed to inhibit $50 \%$ of HIV. $\mathrm{IC}_{50} \mathrm{FC}$ was calculated as the ratio $\mathrm{I} \mathrm{C}_{50}$ for resistant virus $/ \mathrm{IC} \mathrm{C}_{50}$ for wild type virus. MPI (maximum percent inhibition) was calculated as described in Methods. 
Table 3 Summary of susceptibility and phenotypic properties of V2, V3 and V4 single, double and triple mutants against vicriviroc

\begin{tabular}{llllll}
\hline Loop & Mutant & Tropism & IC $_{50}$ & $\begin{array}{l}\text { IC } \\
\text { F0 }\end{array}$ & MPI \\
& & & \multicolumn{3}{c}{ FC increase } \\
\hline Parental clone & Wild type & R5 & $1.56 \mathrm{nM}$ & - & - \\
V2 & V169M & R5 & $1.61 \mathrm{nM}$ & 1.03 & $100 \%$ \\
V3 & L317W & R5 & $1.90 \mathrm{nM}$ & 1.21 & $100 \%$ \\
V4 & I408T & R5 & $3.16 \mathrm{nM}$ & 2.02 & $94 \%$ \\
V2-V3 & V169M & R5 & $1.56 \mathrm{nM}$ & 1.57 & $100 \%$ \\
& L317W & & & & \\
V2-V4 & V169M & R5 & $1.62 \mathrm{nM}$ & 1.03 & $96 \%$ \\
& I408T & & & & \\
V3-V4 & L317W & R5 & $1.68 \mathrm{nM}$ & 1.07 & $93 \%$ \\
& I408T & & & & \\
V2-V3-V4 & V169M & R5 & $1.59 \mathrm{nM}$ & 1.01 & $94 \%$ \\
& L317W & & & & \\
& I408T & & & & \\
\hline
\end{tabular}

$\mathrm{IC}_{50}, 50 \%$ effective concentration or concentration needed to inhibit $50 \%$ of HIV. $I C_{50}$ FC was calculated as the ratio $I C_{50}$ for resistant virus/ $/ C_{50}$ for wild type virus. MPI (maximum percent inhibition) was calculated as described in Methods.

occurred only in viruses resistant to MVC in passage experiments. It was a change from medium size and hydrophobic I to medium size and polar $\mathrm{T}$. The mutation conferred resistance to MVC and was linked with cross-resistance to $\mathrm{VCV}$, as indicated by MPI values. Although cross-resistance was observed for $\mathrm{VCV}$, the virus was more resistant to MVC. Cross-resistance is commonly seen among CCR5 inhibitors [5]. Even if VCV had a favorable resistance profile, its virological activity was sub-optimal in phase II and III studies, possibly because of its pharmacokinetic profile and the dose chosen [5,24]. Tilton et al. [25] analyzed viruses from 11 treatment-experienced patients in whom virologic failure occurred on regimens containing MVC and noted that resistance was dependent upon mutations within the V3 loop which was only modulated by additional mutations in the V4 loop. The V4 mutations consisted of D407G and loss of a glycosylation site at residue 386 .

Platt et al. [26] described HIV-1 CCR5 $(\Delta \mathrm{Nt})$-adapted mutants of the JR-CSF strain that had mutations in regions V3, V2 and C3 with 4 mutations in the V4 loop: N403S, N403K, T405A, and T405N. They concluded that loss of $\mathrm{N}$-glycan at position 403 helps to convert the HIV-1 env into a hairpin-trigger form that no longer requires strong interactions with both the CCR5 amino terminus and ECL2 but efficiently targets either site alone [27-32]. Based on these models, we could postulate that the presence of $\mathrm{T}$ in position 408 of the V4 loop could alter the quaternary structure of the gp120-gp41 trimeric complex, eliciting conformational transition from a high-energy to a low-energy state (fusogenic conformation), enhancing membrane fusion, and promoting the next step in the virus entry pathway. Since positions 403 and 408 of the V4 loop are close proximity, we imagine that the resistance conferred by I408T mutation in our study could alter the quaternary structure of the HIV-1

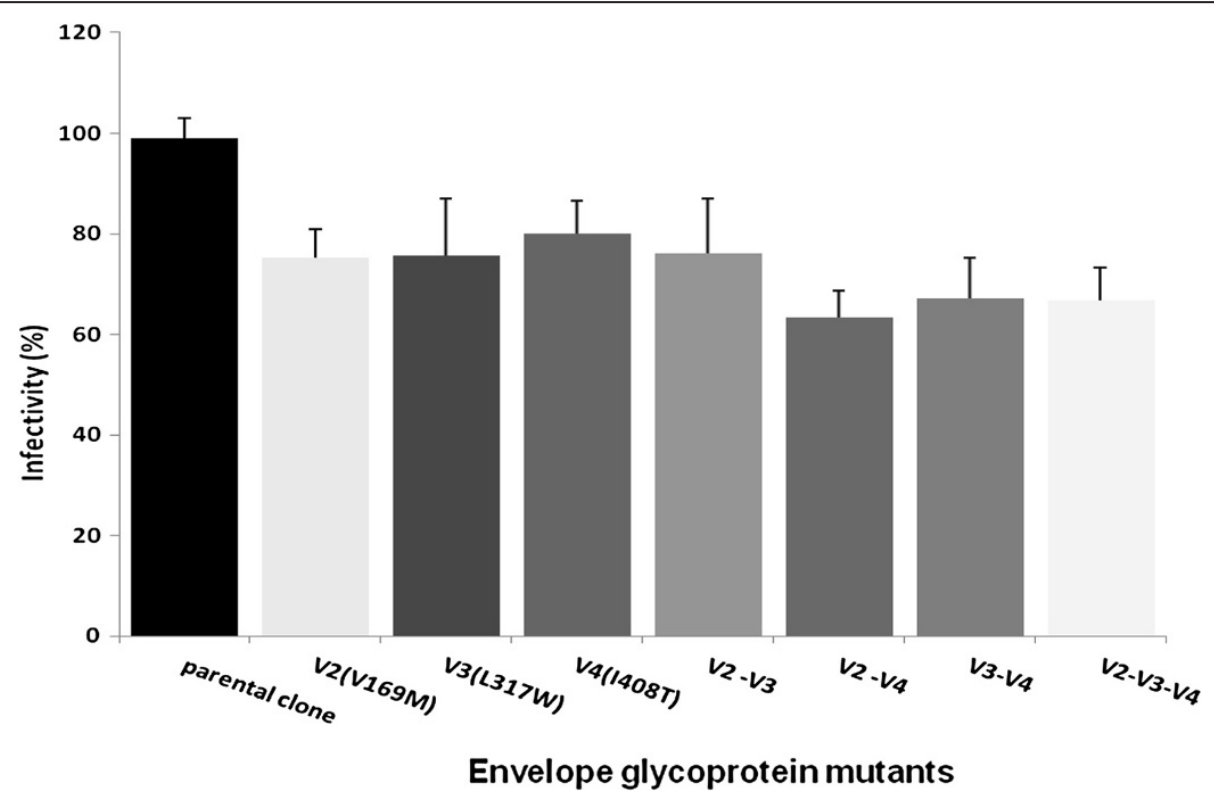

Figure 2 Effect of Env glycoprotein mutations on infectivity of the HIV-1 strain CC1/85. U87-CD4-CCR5 cells were infected with HIV-1 Env pseudoviruses bearing parental clone (wild type) or Env single mutants V169M, L317W, I408T, double mutants V169M/L317W, V169M/l408T, L317W/I408T and the triple mutant V169M/L317W/4408T. Means from 3 independent experiments performed in triplicate are reported. Pseudovirus infectivity was calculated as RLU of the mutant/RLU of the wild type $\times 100 \%$. 
Env, thus sterically masking the glycosylation site in position 403.

Mutations in V2, C3 and gp41 have also been observed in CC1/85-derived, MVC-resistant viruses [14]. The mutation V169M, identified in our study, was also found by Marozsan et al. in VCV escape mutant, which was resistant to $\mathrm{VCV}$, but they did not investigate the contribution of each individual mutation to the resistant phenotype [15]. Recently, Thielen et al. [33] demonstrated that mutations at position 169 of the V2 loop can serve in HIV-1 co-receptor prediction. In their study, the mutation V169T showed strong association with CXCR4 usage while V169K was coupled with CCR5 usage. In another study, the mutation V169K in V2 was predictive of the R5 phenotype [34]. Our results disclosed that mutant V169M exploits R5 exclusively as a co-receptor. V169M mutation was coupled with reduced infectivity but not with a resistant phenotype. Unexpectedly, our triple mutant was less infective than single and double mutants as the emergence of mutations throughout gp120 and gp41 indicated general envelope gene (env) re-arrangement to compensate for decreased replicative capacity $[35,36]$.

\section{Conclusions}

Several mutations outside the V3 loop were shown to contribute to CCR5 inhibitor resistance. Our results showed that I408T, L317W/I408T and V169M/L317W/ I408T mutants had the highest impact on MVC susceptibility, mostly due to I408T in V4. This mutation could lower the activation energy needed to enable gp41 to undergo the next conformational changes and acquire a more stable low-energy state. All mutants retained the CCR5 co-receptor, supporting the concept that resistant viruses maintained the ability to use inhibitor-bound CCR5, depending on co-receptor density on the cellular surface and the degree of CCR5 co-receptor occupancy by drugs.

\section{Methods}

\section{Compounds, cells and viruses}

MVC and VCV were obtained from ViroChem Inc. Canada, now Vertex Canada Inc., reconstituted in DMSO $100 \%$ (Sigma, St. Louis, MO, USA) and diluted in RPMI 1640 culture medium (Sigma). Peripheral blood mononuclear cells (PBMCs) from 3 HIV-negative donors were isolated by Ficoll-Paque gradient separation (Sigma), stimulated for 3 days with phytohemagglutinin (Sigma) at $1 \mathrm{mg} / \mathrm{ml}$ and re-suspended at $2 \times 10^{6}$ cells $/ \mathrm{ml}$ in RPMI 1640 supplemented with $20 \%$ heat-inactivated fetal calf serum (FCS, Invitrogen, Carlsbad, CA, USA), $50 \mathrm{U} / \mathrm{ml}$ penicillin (Invitrogen), $50 \mu \mathrm{g} / \mathrm{ml}$ streptomycin (Invitrogen)., $2 \mathrm{mM}$ L-glutamine, $10 \mathrm{mM}$ hydroxyethylpiperazine N-2 ethanesulfonic acid (HEPES) and $1 \mu \mathrm{g} / \mathrm{ml}$ interleukin-2 (Hoffmann-La Roche, Nutley, NJ, USA) in 6-well plates (Becton Dickinson, Lincoln Park, N.J USA.). 293T cells were cultured in Dulbecco's modified Eagle medium (DMEM) and supplemented with 10\% FCS, $100 \mathrm{U} / \mathrm{ml}$ penicillin and $0.1 \mathrm{mg}$ of streptomycin $/ \mathrm{ml}$ U87CD4CXCR4 and U87CD4CCR5 cells were cultured in DMEM and supplemented with 10\% FCS, $100 \mathrm{U} / \mathrm{ml}$ of penicillin, $0.1 \mathrm{mg}$ of streptomycin $/ \mathrm{ml}, 1$ $\mu \mathrm{g} / \mathrm{mL}$ of puromycin (Sigma) and $300 \mu \mathrm{g}$ of G418 (Invitrogen). All cells were maintained at $37^{\circ} \mathrm{C}$ and $5 \%$ $\mathrm{CO}_{2}$. Cells and the viruses $\mathrm{BaL}$ and $\mathrm{CC1} / 85$ (R5 tropic), III B (X4-tropic) and 85.6 (dual-tropic) were obtained from the National Institutes of Health.

\section{Generation of HIV-1 mutants resistant to MVC and VCV by in vitro passage in PBMCs}

$2 \times 10^{6} \mathrm{PBMCs} / \mathrm{ml}$ were infected with 3,000 tissue culture infective doses $50 \%$ of $\mathrm{BaL}$ and $\mathrm{CC} 1 / 85$ viruses, in a final volume of $2 \mathrm{ml}$ containing sub-inhibitory concentrations of $0.04 \mathrm{nM} \mathrm{MVC}$ and $0.02 \mathrm{nM} \mathrm{VCV}$. Every 7 days, the culture supernatant was passaged onto fresh cells with MVC- and VVC-containing medium, and virus growth was monitored by enzyme-linked immunosorbent assay (ELISA) to measure supernatant p24 levels (Perkin-Elmer, Norwalk, CT, USA). MVC and VCV were added every 4 days at concentrations depending on p24 levels. MVC and VCV control passages were set up in parallel. Full length sequences of gp120 and gp 41 were obtained for different time points, and mutations from resistant viruses were identified. Resistance was defined as MPI $<95 \%$ or $\geq 3$-FC in $\mathrm{IC}_{50}$. Viral tropism was predicted according to $11 / 25$ and net charge rules [37] as well as PSSM $_{\mathrm{X} 4 \mathrm{R} 5}[11,12]$ and geno2pheno co-receptor algorithms [13].

\section{Amplification and sequence analysis of the HIV env gene from selected time-points}

HIV-1 RNA was extracted from culture supernatants with QIAmp Viral RNA mini-kit (Qiagen, Mississauga, ON, Canada). Full-length gp160 was amplified with reverse transcription -polymerase chain reaction (RT-PCR) primers (5' NewFHindIII GGCCAAGCTTATGAGAGT GACGGAGATCAG and 5' YW15XhoI GGCCCTCGA GTTATCCAGTCCCCCCTTTTC), followed by nested polymerase chain reaction (PCR) with primers (5' NewFHindIII GGCCAAGCTTATGAGAGTGACGGAG ATCAG and 5' YW16XhoI GGCCCTCGAGTTATTTT GACCACTTGCCAC). PCR products were separated on $1 \%$ agarose gel and purified with QIAprep Spin Miniprep Kit (Qiagen). Sequencing was undertaken at the GénomeQuébec sequencing facilities in a $3730 \times 1$ DNA analyser from Applied Biosystems (McGill University and the Genome Quebec Innovation Centre, Montreal, QC, Canada) with Sequencer 4.7 (Gene Code Software 
Corporation, Ann Arbor, MI, USA) and aligned by ClustalW version 1.83 [10]. Nucleotide sequences of CC1/85 have been deposited in GenBank under accession numbers JQ924495 (start), JQ924496 (controlP4), JQ924497 (MVCP4), JQ924498 (VCVP4), JQ924499 (controlP16), JQ92450 (MVCP16), and JQ924501 (VCVP16).

\section{Site-directed mutagenesis}

Primers were designed with the Stratagene's web-based QuikChange ${ }^{\circ}$ Primer Design Program [38]. Mutagenesis procedures were carried out according to an overlappingextension PCR-based procedure $[39,40]$.

\section{Cloning and pseudovirus construction}

The expression plasmid pcDNA3.1day/V5HisTOPO (Invitrogen) and Env PCR products of CC1/85, BaL, III $\mathrm{B}, 85.6$ viruses and selected mutants (V169M in V2, L317W in V3 and I408T in V4) in single, double and triple combinations were digested with HindIII and Xho1 restriction enzymes (Invitrogen), purified and ligated with T4 DNA Ligase (Invitrogen). The ligation product was transformed into E. coli TOP10 competent cells. Pseudoviruses were produced by co-transfection of Env expression plasmid and backbone pNL4-3. Luc.E-R-, as described previously [41,42]. Pseudovirus stocks were normalized with p24 ELISA prior to testing infectivity. Assays were performed with $25 \mathrm{ng}$ of p24 per well.

\section{Luciferase assays to determine infectivity and tropism}

A luminescence assay using U87CD4CXCR5 and U87CD4CXCR4 cells was used to measure infectivity and tropism. On the day prior to infection, $1 \times 10^{6}$ cells per well were seeded in 96-well plates. On the day of infection, MVC and AMD 3100 were added to wells designated for treatment with an inhibitor and incubated for $1 \mathrm{~h}$ at $37^{\circ} \mathrm{C}$ prior to infection. $50 \mu \mathrm{l}$ of normalized pseudovirus stocks were added in each well. The plates were incubated for 2 days at $37^{\circ} \mathrm{C}$ with $5 \% \mathrm{CO}_{2}$. The medium was removed, and $100 \mu \mathrm{l}$ of lysis buffer (Promega Inc., Madison, WI, USA) was added to each well for $30 \mathrm{~min}$. Then, $100 \mu \mathrm{l}$ of luciferase assay reagent (Promega) was added immediately prior to reading the plates in a luminometer (Tecan, Morrisville, NC, USA). Luciferase activity was recorded as relative light units (RLU). Viral entry was determined as percent reduction of viral infectivity compared to the controls. All experiments were performed in duplicate. $\mathrm{IC}_{50}$ - $\mathrm{FC}$ was calculated as the ratio of $\mathrm{IC}_{50}$ for resistant virus $/ \mathrm{IC}_{50}$ for wild type virus. MPI was calculated as [1- (RLU in the presence of drug/RLU in the absence of drug)] $\times 100$, and infectivity as RLU of the mutant/RLU of the wild type $x$ $100 \%$. Inhibition curves were generated by GraphPad Prism software (San Diego, CA, USA).

\section{Additional file}

Additional file 1: The Env region of laboratory-adapted $\mathrm{BaL}$ virus after 16 passages in the presence of sub-inhibitory VCV concentrations.

\section{Abbreviations}

(HIV-1): Human immunodeficiency virus-1 entry; (V3): Variable domain 3; $\left(\mathrm{IC}_{50}\right)$ : Half maximal inhibitory concentration; (Env): Envelope protein; (CCR5): C-C chemokine receptor 5; (CXCR4): C-X-C chemokine receptor 4; (MVC): Maraviroc; (VCV): Vicriviroc; (MPI): As maximum percent inhibition; (FC): Measuring fold-change; (env): Envelope gene.

\section{Competing interests}

$\mathrm{CT}$ is the Pfizer/University of Montreal Chair in HIV Translational Research and a scholar from Fonds de la recherche en santé du Québec. The other authors declare no conflicts of interest.

\section{Authors' contributions}

OA-M participated in study conception and design, data collection, analysis and interpretation as well as manuscript drafting; AC supervised the study, analyzed and interpreted the data, and reviewed the manuscript; YW participated in study conception and design; $\mathrm{AH}$ participated in data collection; MS participated in data collection, analysis and interpretations; CT participated in study conception and design, data analysis and interpretation, study supervision, and manuscript review. All authors read and approved the final manuscript.

\section{Acknowledgments}

We thank Dr. Andrés Finzi for reading our manuscript and offering valuable suggestions, Dr. Amal Nadiri for discussions on and assistance with mutagenesis, and the NIH AIDS Research and Reference Reagent Program for the viruses, the backbone pNL4-3. Luc.E-R- and U87CD4CXCR4, U87CD4CCR5 and 293T cells

\section{Funding}

This work was supported by the Réseau Fonds de la recherche en santé du Québec (FRSQ)-SIDA.

\section{Author details}

${ }^{1}$ Centre de recherche, Centre hospitalier de I'Université de Montréal (CRCHUM), Montréal, QC, Canada. ${ }^{2}$ Department of Microbiology and Immunology, Faculty of Medicine, Université de Montréal, Montréal, QC, Canada. ${ }^{3}$ Laboratoire de santé publique du Québec/Institut national de santé publique du Québec (INSPQ), Québec, Canada. ${ }^{4} \mathrm{CRCHUM} \mathrm{-} \mathrm{Hôtel-Dieu,}$ Pavillon Jeanne-Mance, bureau 7-355, 3840 rue St-Urbain, Montréal, QC H2W 1T8, Canada.

Received: 4 April 2013 Accepted: 4 June 2013

Published: 7 June 2013

\section{References}

1. Chen B, Vogan EM, Gong H, Skehel JJ, Wiley DC, Harrison SC: Structure of an unliganded simian immunodeficiency virus gp120 core. Nature 2005, 433:834-841.

2. Kwong PD, Wyatt R, Robinson J, Sweet RW, Sodroski J, Hendrickson WA Structure of an HIV gp120 envelope glycoprotein in complex with the CD4 receptor and a neutralizing human antibody. Nature 1998, 393:648-659.

3. Pfaff JM, Wilen CB, Harrison JE, Demarest JF, Lee B, Doms RW, Tilton JC: HIV-1 resistance to CCR5 antagonists associated with highly efficient use of CCR5 and altered tropism on primary CD4+ T cells. J Virol 2010, 84:6505-6514.

4. Tilton JC, Doms RW: Entry inhibitors in the treatment of HIV-1 infection. Antiviral Res 2010, 85:91-100.

5. Berro R, Klasse PJ, Moore JP, Sanders RW: V3 determinants of HIV-1 escape from the CCR5 inhibitors Maraviroc and Vicriviroc. Virology 2012, 427:158-165. 
6. Kondru R, Zhang J, Ji C, Mirzadegan T, Rotstein D, Sankuratri S, Dioszegi M: Molecular interactions of CCR5 with major classes of small-molecule anti-HIV CCR5 antagonists. Mol Pharmacol 2008, 73:789-800.

7. Strizki JM, Tremblay C, Xu S, Wojcik L, Wagner N, Gonsiorek W, Hipkin RW, Chou CC, Pugliese-Sivo C, Xiao Y, et al: Discovery and characterization of vicriviroc (SCH 417690), a CCR5 antagonist with potent activity against human immunodeficiency virus type 1. Antimicrob Agents Chemother 2005, 49:4911-4919.

8. McNicholas PM, Mann PA, Wojcik L, Phd PQ, Lee E, McCarthy M, Shen J, Black TA, Strizki JM: Mapping and characterization of vicriviroc resistance mutations from HIV-1 isolated from treatment-experienced subjects enrolled in a phase II study (VICTOR-E1). J Acquir Immune Defic Syndr 2011, 56:222-229.

9. Roche M, Jakobsen MR, Sterjovski J, Ellett A, Posta F, Lee B, Jubb B, Westby M, Lewin SR, Ramsland PA, et al: HIV-1 escape from the CCR5 antagonist maraviroc associated with an altered and less-efficient mechanism of gp120-CCR5 engagement that attenuates macrophage tropism. J Virol 2011, 85:4330-4342.

10. McNicholas P, Wei Y, Whitcomb J, Greaves W, Black TA, Tremblay CL, Strizki JM: Characterization of emergent HIV resistance in treatment-naive subjects enrolled in a vicriviroc phase 2 trial. J Infect Dis 2010, 201:1470-1480.

11. Jensen MA, Li FS, van't Wout AB, Nickle DC, Shriner D, He HX, McLaughlin S, Shankarappa R, Margolick JB, Mullins JI: Improved coreceptor usage prediction and genotypic monitoring of R5-to- $\mathrm{X} 4$ transition by motif analysis of human immunodeficiency virus type 1 env V3 loop sequences. J Virol 2003, 77:13376-13388.

12. Jensen $M A$, Coetzer $M$, van't Wout $A B$, Morris $L$, Mullins Jl: A reliable phenotype predictor for human immunodeficiency virus type 1 subtype C based on envelope V3 sequences. J Virol 2006, 80:4698-4704.

13. Lengauer $T$, Sander $O$, Sierra $S$, Thielen A, Kaiser R: Bioinformatics prediction of HIV coreceptor usage. Nat Biotechnol 2007, 25:1407-1410.

14. Westby M, Smith-Burchnell C, Mori J, Lewis M, Mosley M, Stockdale M, Dorr P, Ciaramella G, Perros M: Reduced maximal inhibition in phenotypic susceptibility assays indicates that viral strains resistant to the CCR5 antagonist maraviroc utilize inhibitor-bound receptor for entry. J Virol 2007, 81:2359-2371

15. Marozsan AJ, Kuhmann SE, Morgan T, Herrera C, Rivera-Troche E, Xu S, Baroudy BM, Strizki J, Moore JP: Generation and properties of a human immunodeficiency virus type 1 isolate resistant to the small molecule CCR5 inhibitor, SCH-417690 (SCH-D). Virology 2005, 338:182-199.

16. Trkola A, Kuhmann SE, Strizki JM, Maxwell E, Ketas T, Morgan T, Pugach P, Xu S, Wojcik L, Tagat J, et al: HIV-1 escape from a small molecule, CCR5-specific entry inhibitor does not involve CXCR4 use. Proc Natl Acad Sci USA 2002, 99:395-400.

17. Kuhmann SE, Pugach P, Kunstman KJ, Taylor J, Stanfield RL, Snyder A, Strizki JM, Riley J, Baroudy BM, Wilson IA, et al: Genetic and phenotypic analyses of human immunodeficiency virus type 1 escape from a small-molecule CCR5 inhibitor. J Virol 2004, 78:2790-2807.

18. Roche M, Jakobsen MR, Ellett A, Salimiseyedabad H, Jubb B, Westby M, Lee B, Lewin SR, Churchill MJ, Gorry PR: HIV-1 predisposed to acquiring resistance to maraviroc (MVC) and other CCR5 antagonists in vitro has an inherent, low-level ability to utilize MVC-bound CCR5 for entry. Retrovirology 2011, 8:89.

19. Zolla-Pazner S, Cardozo T: Structure-function relationships of HIV-1 envelope sequence-variable regions refocus vaccine design. Nat Rev Immunol 2010, 10:527-535.

20. Anastassopoulou CG, Ketas TJ, Klasse PJ, Moore JP: Resistance to CCR5 inhibitors caused by sequence changes in the fusion peptide of HIV-1 gp41. Proc Natl Acad Sci USA 2009, 106:5318-5323.

21. Yu X, Yuan L, Huang Y, Xu W, Fang Z, Liu S, Shao Y, Jiang S, Ma L: Susceptibility of HIV-1 Subtypes B', CRF07_BC and CRF01_AE that Are Predominantly Circulating in China to HIV-1 Entry Inhibitors. PLOS One 2011, 6:e17605.

22. Yuan $Y$, Maeda Y, Terasawa H, Monde K, Harada S, Yusa K: A combination of polymorphic mutations in V3 loop of HIV-1 gp120 can confer noncompetitive resistance to maraviroc. Virology 2011, 413:293-299.

23. Munoz-Nieto M, Perez-Alvarez L, Thomson M, Garcia V, Ocampo A, Casado G, Delgado E, Miralles C, de Parga EV, Sierra M, et al: HIV type 1 intersubtype recombinants during the evolution of a dual infection with subtypes B and G. AIDS Res Hum Retroviruses 2008, 24:337-343.
24. Wilkin TJ, Gulick RM: CCR5 antagonism in HIV infection: current concepts and future opportunities. Annu Rev Med 2012, 63:81-93.

25. Tilton JC, Wilen CB, Didigu CA, Sinha R, Harrison JE, Agrawal-Gamse C, Henning EA, Bushman FD, Martin JN, Deeks SG, Doms RW: A maravirocresistant HIV-1 with narrow cross-resistance to other CCR5 antagonists depends on both $\mathrm{N}$-terminal and extracellular loop domains of drugbound CCR5. J Virol 2010, 84:10863-10876.

26. Platt EJ, Shea DM, Rose PP, Kabat D: Variants of human immunodeficiency virus type 1 that efficiently use CCR5 lacking the tyrosine-sulfated amino terminus have adaptive mutations in gp120, including loss of a functional N-glycan. J Virol 2005, 79:4357-4368.

27. Wyatt R, Kwong PD, Desjardins E, Sweet RW, Robinson J, Hendrickson WA, Sodroski JG: The antigenic structure of the HIV gp120 envelope glycoprotein. Nature 1998, 393:705-711.

28. Wyatt R, Sodroski J: The HIV-1 envelope glycoproteins: fusogens, antigens, and immunogens. Science 1998, 280:1884-1888.

29. Xiang SH, Finzi A, Pacheco B, Alexander K, Yuan W, Rizzuto C, Huang CC, Kwong PD, Sodroski J: A V3 loop-dependent gp120 element disrupted by CD4 binding stabilizes the human immunodeficiency virus envelope glycoprotein trimer. J Virol 2010, 84:3147-3161.

30. Cormier EG, Dragic T: The crown and stem of the V3 loop play distinct roles in human immunodeficiency virus type 1 envelope glycoprotein interactions with the CCR5 coreceptor. J Virol 2002, 76:8953-8957.

31. Cormier EG, Tran DN, Yukhayeva L, Olson WC, Dragic T: Mapping the determinants of the CCR5 amino-terminal sulfopeptide interaction with soluble human immunodeficiency virus type $1 \mathrm{gp} 120-\mathrm{CD} 4$ complexes. J Virol 2001, 75:5541-5549.

32. Huang CC, Lam SN, Acharya P, Tang M, Xiang SH, Hussan SS, Stanfield RL, Robinson J, Sodroski J, Wilson IA, et al: Structures of the CCR5 N terminus and of a tyrosine-sulfated antibody with HIV-1 gp120 and CD4. Science 2007, 317:1930-1934.

33. Thielen A, Sichtig N, Kaiser R, Lam J, Harrigan PR, Lengauer T: Improved prediction of HIV-1 coreceptor usage with sequence information from the second hypervariable loop of gp120. J Infect Dis 2010, 202:1435-1443.

34. Monno L, Saracino A, Scudeller L, Punzi G, Brindicci G, Altamura M, Lagioia A, Ladisa N, Angarano G: Impact of mutations outside the $\mathrm{V} 3$ region on coreceptor tropism phenotypically assessed in patients infected with HIV-1 subtype B. Antimicrob Agents Chemother 2011, 55:5078-5084.

35. Gonzalez-Ortega E, Ballana E, Badia R, Clotet B, Este JA: Compensatory mutations rescue the virus replicative capacity of VIRIP-resistant HIV-1. Antiviral Res 2011, 92:479-483.

36. Lucas GM: Antiretroviral adherence, drug resistance, viral fitness and HIV disease progression: a tangled web is woven. J Antimicrob Chemother 2005, 55:413-416.

37. Poveda E, Alcami J, Paredes R, Cordoba J, Gutierrez F, Llibre JM, Delgado R, Pulido F, Iribarren JA, Garcia Deltoro M, et al: Genotypic determination of HIV tropism - clinical and methodological recommendations to guide the therapeutic use of CCR5 antagonists. AIDS Rev 2010, 12:135-148.

38. The stratagene's web-based Primer Design Program QuikChange ${ }^{\circledast}$ available at website at [http://www.genomics.agilent.com/primerDesignProgram.jsp]

39. Kanoksilapatham, GJM W, Robb FT: Directed-Mutagenesis and Deletion Generated through an Improved Overlapping-Extension PCR Based Procedure. Silpakorn U Sci Tech J Vol 2007, 1:7-12.

40. Aiyar $A$, Xiang $Y$, Leis J: Site-directed mutagenesis using overlap extension PCR. Methods Mol Biol 1996, 57:177-191.

41. Connor Rl, Sheridan KE, Lai C, Zhang L, Ho DD: Characterization of the functional properties of env genes from long-term survivors of human immunodeficiency virus type 1 infection. J Virol 1996, 70:5306-5311.

42. Singh A, Page T, Moore PL, Allgaier RL, Hiramen K, Coovadia HM, Walker BD, Morris L, Ndung'u T: Functional and genetic analysis of coreceptor usage by dualtropic HIV-1 subtype C isolates. Virology 2009, 393:56-67.

doi:10.1186/1742-6405-10-15

Cite this article as: Asin-Milan et al:: Mutations in variable domains of the HIV-1 envelope gene can have a significant impact on maraviroc and vicriviroc resistance. AIDS Research and Therapy 2013 10:15. 